\title{
Pulsar B0531 + 21 in the Crab Nebula
}

\author{
Boris Ja. Losovsky \\ Physical Institute of the Russian Academy of Science, Pushchino Radio Astronomy Observatory, Moscow, Russia \\ Email: blos@prao.ru
}

How to cite this paper: Losovsky, B.Ja. (2021) Pulsar B0531 + 21 in the Crab Nebula. International Journal of Astronomy and Astrophysics, 11, 470-480. https://doi.org/10.4236/ijaa.2021.114024

Received: September 15, 2021 Accepted: November 12, 2021 Published: November 15, 2021

Copyright $\odot 2021$ by author(s) and Scientific Research Publishing Inc. This work is licensed under the Creative Commons Attribution International License (CC BY 4.0).

http://creativecommons.org/licenses/by/4.0/

\begin{abstract}
The Crab Nebula and its pulsar PSR B0531 +21 is the space laboratory for the study of fundamental physical processes. From the beginning of the current century up to date, the giant pulses of the pulsar in the Crab Nebula are regularly observed on the Large Phased Array of the Pushchino Radio Astronomy Observatory of the Lebedev Physical Institute at a frequency $111 \mathrm{MHz}$. The connection between the scattering time scale of radio pulses and the dispersion measure of the pulsar, which was established earlier together with Jodrell Bank, is confirmed. The observed variations in the scattering of radio pulses and their partial correlation with the dispersion measure are explained by the eclipse of the pulsar by dense plasma clouds with fluctuations in the electron density significantly exceeding the corresponding fluctuations in the interstellar medium. The question of a possible connection between period failers (glitches), dispersion measure variations, radio pulses scattering and gamma-ray flares is discussed.
\end{abstract}

\section{Keywords}

Pulsar, Giant Pulses, Dispersion Measure, Braking Index, Glitches, Gamma-Ray Flares

\section{Introduction}

The Crab Nebula was first observed in 1731 by the English physicist and amateur astronomer John Bevis. It is included in the catalog of nebulae compiled by Charles Monsieur in 1758 as object M1. The Crab Nebula was so named by Lord Ross in 1844. Observing the nebula with high resolution, he noticed a structure similar to a crab claw. It is believed that it is a remnant of the hydrogen-rich type II supernova explosion, observed in China in 1054. In 1949, radio astronomers Bolton, Stanley, and Slee identified the Crab Nebula as a radio source. Buhler and Blandford [1] write: "Due to its high luminosity $L \approx 1.3 \times 10^{38} \mathrm{erg} \cdot \mathrm{s}^{-1}$ (Hester 2008) and its proximity of $\sim 2 \mathrm{kpc}$ (Trimble 1973), the Crab can be studied in 
great detail. It is therefore one of our prime laboratories to study non-thermal processes in the Universe. Many discoveries have been made in the Crab and then been seen in other non-thermal sources (including active galactic nuclei, A gamma ray bursts and X-ray binaries) such as polarized synchrotron radiation or pulsed optical emission (Shklovsky 1953, Cocke et al. 1969). True to this legacy two remarkable discoveries have been made in the last years. Very high-energy (VHE \& $100 \mathrm{GeV}$ ) gamma-ray emission has been detected from the pulsar, and high-energy (HE \& $100 \mathrm{MeV}$ ) gamma-ray flares have been discovered from the nebula. These phenomena have not been observed in any other pulsar wind nebula to date".

The Crab Nebula pulsar PSR B0531 + 21 (Crab Pulsar) was discovered by Staeilin and Reifenstein in 1968 as a radio source of giant pulses (GPs) ${ }^{1}$, rather than as a periodic source [2]. Fourier analysis did this later. The phenomena of scattering time scale $\tau_{s c}{ }^{2}$ and the dispersion measures (DM) variations of the GPs in the Crab Pulsar were first recorded by Rankin and Counselman [3] from observations in Arecibo in the range of $74-430 \mathrm{MHz}$ in 1969-1971. Analyzing the scattering variations, they concluded that these processes occur mainly within the Crab Nebula. Isaacman and Rankin [4] continued the measurements until 1974. They also found variations in the scattering time scale and the DM, but found no significant relationship between these phenomena.

Since the beginning of the current century and up to the present time, regular observations of GPs from the Crab Pulsar at $111 \mathrm{MHz}$ have been carried out on the Large Phased Array of the Pushchino Radio Astronomy Observatory (LPA PRAO) of the Lebedev Physical Institute. This article systematizes the data obtained during the observation of the Crab Pulsar [5] [6] [7]. In particular, we show that the scattering of GPs of the Crab pulsar depends not only on the DM, but also on the degree of plasma inhomogeneity. The possible relationship between step changes in the rotation speed ("glitch"), the DM variations and scattering time scale is discussed. Results of the search for a connection between gamma-ray flares and disturbances in the pulsar magnetosphere are given.

\section{Observations and Results}

Systematic observations of the Crab Pulsar at a frequency of $111 \mathrm{MHz}$ are carried out on LPA radio telescope from September 2002 up to date. The observations were initially carried out using a 128-channel spectrum analyzer with a channel bandwidth of $20 \mathrm{kHz}$, a total bandwidth $2560 \mathrm{kHz}$, and a readout interval 2.56 ms. Beginning from April 2006 the observations were conducted using 512-channel digital spectrum analyzer with a channel bandwidth of $5 \mathrm{kHz}$ and readout interval $2.56 \mathrm{~ms}$. The linear polarization of E.-W. Has been registered. GPs are analyzed using a special program that allows us to determine the scat-

${ }^{1}$ The working definition of giant pulses is a flux density in a single pulse which is more than 10 times the mean flux density.

${ }^{2}$ The scattering time scale $\tau_{s c}$ in the interstellar medium is measure of the pulse broadening, and dispersion measure DM measures the total electron content between the pulsar and the observer. 
tering time scale pulsar radio pulses by simulating the passage of a pulse through a scattering medium. A theoretical scattering pulse profile was generated by successively convolving a gaussian profile, representing the intrinsic pulse, with: 1) an impulse scattering function of the form $\left.\exp \left(-t / \tau_{s c}\right) ; 2\right)$ a gaussian function representing the effects of interstellar dispersion across the filter bandpass; 3 ) an exponential function representing the post-detector RC time constant. The theoretical profile was least-squares fitted to the observed profile, with $\tau_{s c}$ as the adjustable parameter [8].

The scattering values for each GP were averaged over five days of observations. The mean scattering parameters were compared with dispersion measures derived from data obtained at Jodrell Bank Observatory (JBO) ${ }^{3}$. The monthly JBO data were interpolated to the comparison data.

Preliminary data on the correlation between the variations of scattering time scale $\tau_{s c}$ and the $D M$ were obtained at the LPA PRAO by Kuzmin and Losovsky at a frequency of $111 \mathrm{MHz}$ together with Jordan and Smith from the JBO in 2006-2007 [9]. However, due to the relatively short duration of this coincidence and the shift between the compared phenomena (the DM variations are 30 days late relative to the scattering variations), it was impossible to exclude the randomness of this event. It was necessary to continue the measurements to make sure that the correlation really took place.

In the course of observations in the period 2008-2013, a long non-stationary process was recorded, characterized by abnormally strong scattering time scale $\tau_{\mathrm{sc}}$ reaching up to $115 \mathrm{~ms}$ (Figure 1). We can estimate the size of the massive plasma cloud that eclipsed the pulsar in 2008-2013. It is known that the $D M$ $\left(\mathrm{pc} / \mathrm{cm}^{3}\right)=n_{e}\left(\mathrm{~cm}^{-3}\right) \times D(\mathrm{pc})$, where $n_{e}$-mean electron density and $D$-cloud size. The scattering value at a frequency of $111 \mathrm{MHz}$ in January 2008 with $D M=$ 56.763 was $20 \mathrm{~ms}$. In December 2012, with the $D M=56.855$, the scattering $\tau_{s c}$ was about $115 \mathrm{~ms}$. Electron density of the filament $n_{e}$ according to published data, is about $10^{3} \mathrm{~cm}^{-3}$ [10]. To increase the $D M$ by $0.092 \mathrm{pc} / \mathrm{cm}^{3}$, the cloud size " $D$ " should be $0.092 / 10^{3}=0.92 \times 10^{-4} \mathrm{pc}=2.84 \times 10^{14} \mathrm{~cm}=19$ AU. During the observation period (2002-2020), there were several glitches ${ }^{4}$.

During the same period, a number of Gamma-ray flares $>100 \mathrm{Mev}$ has been detected by Fermi Gamma-ray Space Telescope [11] [12] [13] and AGILE-X-ray and gamma-ray astronomical satellite [14] [15] [16] [17] (See Figure 1: dash-line segments).

Figure 2 shows the statistical relationship between the scattering time scale $\tau_{s c}$ (in milliseconds) and the conditional Crab Nebular dispersion measure, $d m=$ $(D M-56.7) \times 1000$ in $\mathrm{pc} / \mathrm{cm}^{3}$ of the Crab Pulsar at a frequency of $111 \mathrm{MHz}$ in the period 2002-2020. The error of the scattering time scale measurement is estimated at $5 \%$. The relationship between the scattering time scale and dispersion measure is given by expression

${ }^{3}$ http://www.jb.man.ac.uk/pulsar/crab/crab2.txt.

${ }^{4}$ http://www.jb.man.ac.uk/pulsar/glitches/gTable.html. 


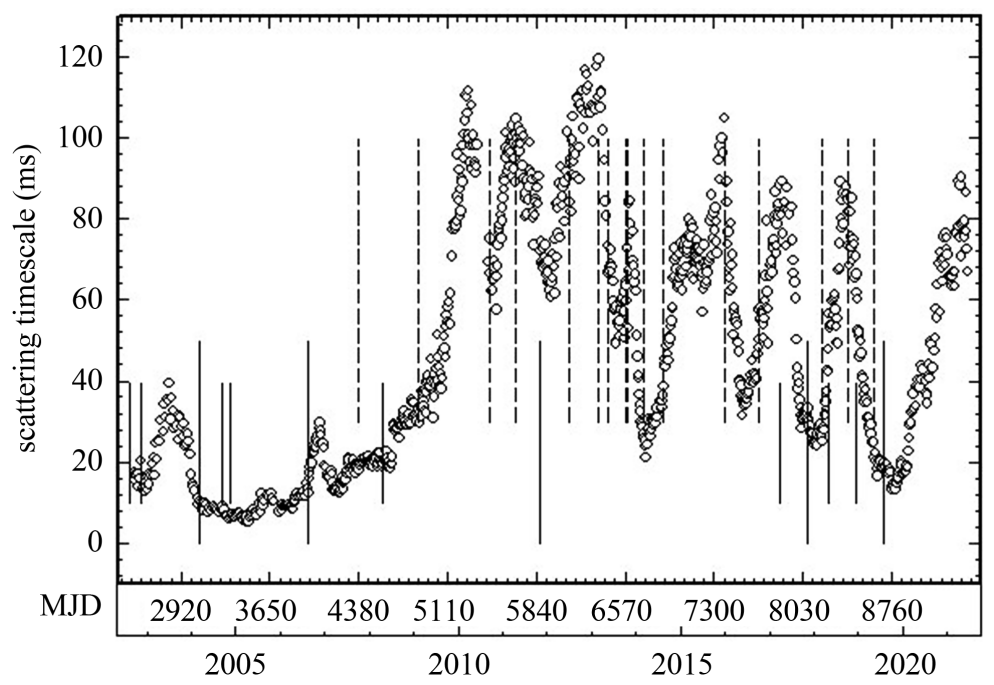

Figure 1. Change in the scattering time scale of the pulsar in the Crab Nebula at a frequency of $111 \mathrm{MHz}$ in the period 2002-2020. Dash-line shows gamma-ray flares. Short segments-strong glitches, shortened segments-weak glitches. On the abscissa axis, the observation epoch in modified Julian days MJD = JD - 2,450,000 and the corresponding years are given.

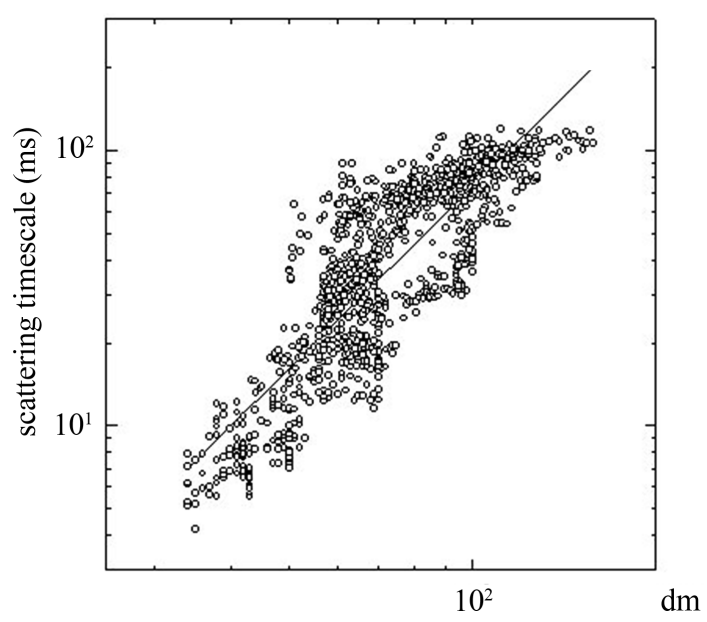

Figure 2. Statistical relationship between the scattering time scale (in milliseconds) $\tau_{s c}$ and the $d m=(D M-56.7) \times 1000 \mathrm{pc} / \mathrm{cm}^{3}$ of the Crab Pulsar at a frequency of $111 \mathrm{MHz}$ in the period 2002-2020.

$$
\lg \left(\tau_{s c}\right)=-2.51(8)+2.19(4) \times \lg (d m) \text { or } \tau_{s c}=C \times d m^{(2.19 \pm 0.04)}
$$

A comparison of the scattering time scale variations and the conditional dispersion measure $d m=(D M-56.7) \times 1000$ confirms their dependence (Figure 3 ). The correlation coefficient is $0.80 \pm 0.05$. The results obtained are in good agreement with measurements of the radio emission of the Crab Pulsar at a frequency of $610 \mathrm{MHz}$ on the 42 -foot radio telescope of the JBO and at a frequency of $1400 \mathrm{MHz}$ on the Lovell radio Telescope. Based on the results of observations in the period from 2008 to 2012, the dependence between the $\tau_{s c}$ and the $D M$ with a correlation coefficient of $0.56 \pm 0.01$ was obtained [18]. 


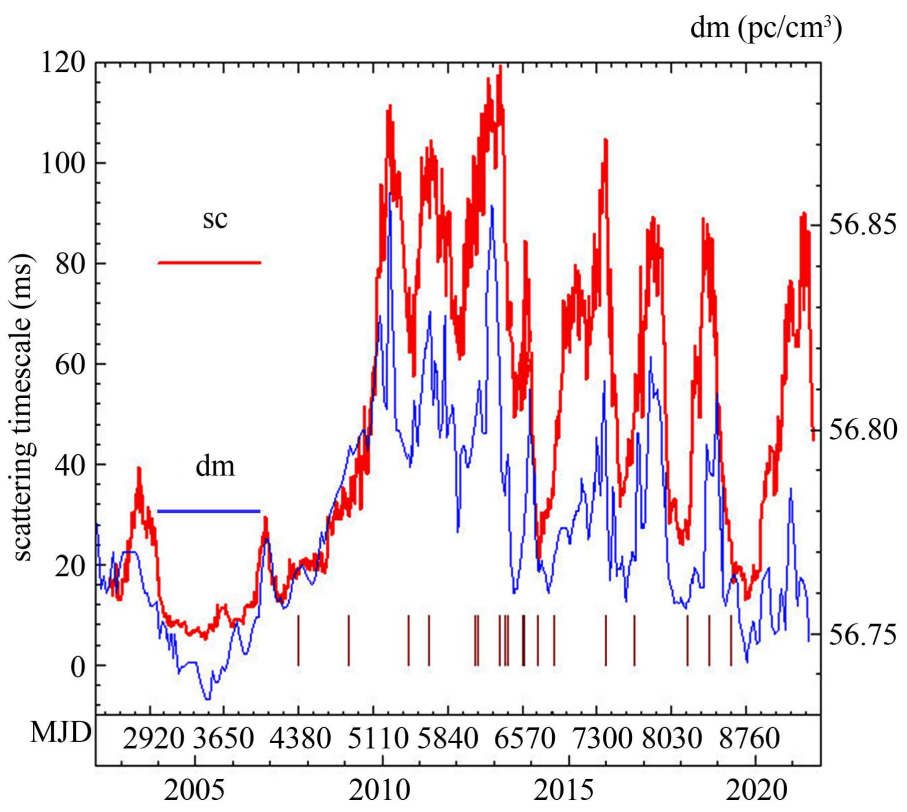

Figure 3. Comparison of the scattering time scale variations in ms (scale on the left, red) and the conditional $d m=(D M-56.7) \times 1000$ (scale on the right, blue). On the abscissa axis-the epoch of observations in modified Julian days MJD = JD - 2,450,000 and the corresponding years. The correlation coefficient is $0.80 \pm 0.05$. Gamma-ray flares $>100$ Mev, detected by Fermi Gamma-ray Space Telescope and AGILE X-ray and gamma-ray astronomical satellite are indicated by brown vertical line [11]-[17].

The scattering time scale $\tau_{s c}$ depends on: the electron density $n_{e}$; the relative inhomogeneity of the medium $\Delta n_{e} / n_{e}$, the dispersion measure $D M$, (or the distance between the source and the observer $d$ ); the frequency of the radio signal $v$ [10].

$$
\tau_{s c} \sim\left(d_{*} \Delta n_{e}\right)^{2} / v^{4}=\left[\left(\Delta n_{e} / n_{e}\right) \times D M\right]^{2} / v^{4}
$$

In an environment, where the parameter $\Delta n_{e} / n_{e}$ is almost constant, the scattering at a given frequency is proportional to the square of the DM:

$$
\tau_{s c} \sim C \times D M^{2}
$$

This is what is observed for most pulsars [19]. The scattering variations in the first approximation are proportional to the variations of $D M$ :

$$
\Delta \tau_{s c} \sim C 1 \times \Delta D M
$$

It follows from the Formula (2) that the scattering can be different with the same $D M$.

In the Crab Nebula, the parameter $\left(\Delta n_{e} / n_{e}\right)$ is subject to significant changes, so the variations of the $\tau_{s c}$ parameter depend not only on the dispersion measure, but also on the degree of plasma inhomogeneity $\left(\Delta n_{e} / n_{e}\right)$ :

$$
\Delta \tau_{s c} \sim C 1 \times \Delta(D M)+C 2 \times \Delta\left(\Delta n_{e} / n_{e}\right)
$$

The spectrum of electron density fluctuations

$$
F(q)=C_{n}^{2} \times q^{-\alpha},
$$


where $C_{n}^{2}\left(\mathrm{~m}^{-\alpha-3}\right)$ is the turbulence coefficient [20]; $q$-spatial frequency, $q=$ $2 \pi / l$-where $l$ is the character scale of turbulence $\alpha$-spectral index of the electron-density irregularity.

$$
C_{n}^{2}=2 \times 10^{-3} v^{\beta} d^{-\beta / 2} \Delta v^{-(\beta-2) / 2},
$$

where $\beta$-spectral index of pulse broadening $\beta=2 \alpha /(\alpha-2)$.

Here $v$ is the frequency in $\mathrm{GHz}, \Delta v$ is the decorrelation bandwidth in $\mathrm{MHz}, d$ is in kpc.

Measurements of GPs from the Crab Pulsar over a wide range of frequencies from 40 to $2228 \mathrm{MHz}$ show that the frequency dependence of scatter-broadening of the pulses is in agreement with a model with normal Gaussian distribution of the inhomogeneities $(\alpha=\beta=4)$ [21], and

$$
C_{n}^{2}=2 \times 10^{-3} v^{4} d^{-2} \Delta v^{-1} \text {. }
$$

The scattering $\tau_{s c}$ and the decorrelation bandwidth $\Delta v$ are related by the ratio $2 \pi \tau_{s c} \Delta v=1$. In particular, for the frequency $v=111 \mathrm{MHz}$ and $\tau_{s c}=1 /(2 \pi \Delta v)$ turbulence level

$$
C_{n}^{2}=0.00198 \tau_{s c} d^{-2},
$$

where $\tau_{s c}$ is in milliseconds.

The period from 2002 to 2007 with a small dispersion measure and small scattering variations is conditionally considered as quiet and the period from 2009 to 2013 with a large dispersion measure and significant scattering variations is considered as active.

We accepted mean scattering parameter $\tau_{s c}=10 \mathrm{~ms}$ for the passive period and $\tau_{s c}=100 \mathrm{~ms}$ for the active period. Error estimated as $20 \%$.

According to Yao et al. [22] distance to Crab pulsar $d=1.311 \mathrm{kpc}(\mathrm{Err}=14 \%)$

In 2002-2007, during a quiet period $C_{n}^{2}=0.0115 \pm 0.0038 \mathrm{~m}^{-7}$. The variations of $D M$ measure $\triangle D M$ were 0.035 , which at a radius of nebular $1.7 \mathrm{pc}$ corresponds to $\Delta n_{e} \sim 0.02 \mathrm{~cm}^{-3}$ and this is consistent with the estimate [18].

If, according to [23], the value of the dispersion measure of the Crab nebula can be taken as $4 \mathrm{pc} / \mathrm{cm}^{3}$, then $n_{e} \sim 2 \mathrm{~cm}^{-3}$ and $\Delta n_{e} / n_{e} \sim 0.01$.

In 2009-2013, during the active period $C_{n}^{2}=0.115 \pm 0.038 \mathrm{~m}^{-7}$. The variations of the $D M$ were $\triangle D M=0.105$, which corresponds to the average value of the density variations $\Delta n_{e} \sim 0.06 \mathrm{~cm}^{-3}$ and $\Delta n_{e} / n_{e} \sim 0.03$.

In the interstellar medium in the direction of the Crab Pulsar [18] $\Delta n_{e}=2 \times$ $10^{-6} \mathrm{~cm}^{-3}$. With $n_{e} \sim 0.03 \mathrm{~cm}^{-3} \Delta n_{e} / n_{e} \sim 1.0 \times 10^{-4}$.

Turbulence in the disturbed region higher than in quiet ones and much higher than in the interstellar medium.

\section{Discussion}

Pulsars gradually slow down as a result of the conversion of rotational energy into the energy of charged particles and electromagnetic waves. In the simple model of pulsar rotation frequency $f$, the first derivative of the rotation frequen- 
cy $\mathrm{d} f \mathrm{~d} t$ and the second derivative of the rotation frequency $\mathrm{d}^{2} f / \mathrm{d} t^{2}$ are used. In this case power-low slowdown is the form $\mathrm{d} f \mathrm{~d} t=-k \times f$. The value of exponent of $f$ is known as braking index $n$. The value of the braking index

$$
n=\left(f \times \mathrm{d}^{2} f / \mathrm{d} t^{2}\right) /(\mathrm{d} f / \mathrm{d} t)^{2}
$$

The braking index $n$ is expected to be 3 in the case of braking by pure dipole radiation and 1 in the case of pulsar wind dominated torque. Deviations from value 3 for Crab pulsar $(n \sim 2.5)$ indicates, than the part of the torque on the pulsar is due to the outflow of particles.

Jumping irregularities in the rotation of the pulsar (glitches) can be of two types: discrete glitches and slow glitches. Both types of glitches cause accelerated rotation of the pulsar, which occurs against the background of secular deceleration of the neutron star. With discrete glitches, the frequency increases suddenly, and then follows an exponential decrease in frequency to the previous value. Slow glitches are associated with slow frequency fluctuations.

The hypothesis about the connection of random irregularities of period with processes in the magnetosphere was firstly expressed Manchester and Taylor in the book "PULSARS" [10]. There are some models, describing the origin of glitches. One model treats glitches as star quakes, caused by the rearrangement of the flattened crust, which tends to become spherical, as the star's rotation slows down. Another model considers a neutron star as a reservoir, filled with a superfluid, the mass of which, when the pulsar's rotation slows down, transmits the angular momentum to the crust, which leads to a glitch [24].

The problem of the relationship between glitches, scattering changes, dispersion measures and gamma-ray bursts is discussed in detail Cadez et al. [25]. They devoted their work to the study of the pulsar in the Crab nebula, using radio data from the Jodrell Bank Observatory from 1998 to 2014 together with optical observations of the pulsar, using the ultra-fast photon counter, mounted on the Copernico telescope at the Astrophysical Observatory in Asiago (Italy) in 2008 and at the Southern European Telescope at La Silla observatory (Chile) in 2009. Analysis of the data showed, that jumps in the braking index are associated with large glitches, when the relative change in the rotational speed of the pulsar exceeds $10^{-8}$. A similar conclusion was obtained by Lyne et al. with the mean value $n=2.34$ [26]. Different braking index values 2.45 and 2.57 are also reported between glitches by Kou and Tong [27].

The delay in the variations of the dispersion measure relative to the variations of the braking index is $\sim 1010$ days and is explained by the time of ionization of the nebula by the pulsar wind (Figure 4).

The model of Cadez et al. [25] suggests, that glitches and subsequent variations in the braking index are caused by instability in the magnetosphere, which changes the configuration of the magnetic field and the currents in the plasma, through which the pulsar interacts with the nebula. Perturbations in the pulsar's magnetosphere can lead to glitches and jumps in the braking index, to the ejection of charged particles into the nebula, to the formation of filaments and to an 


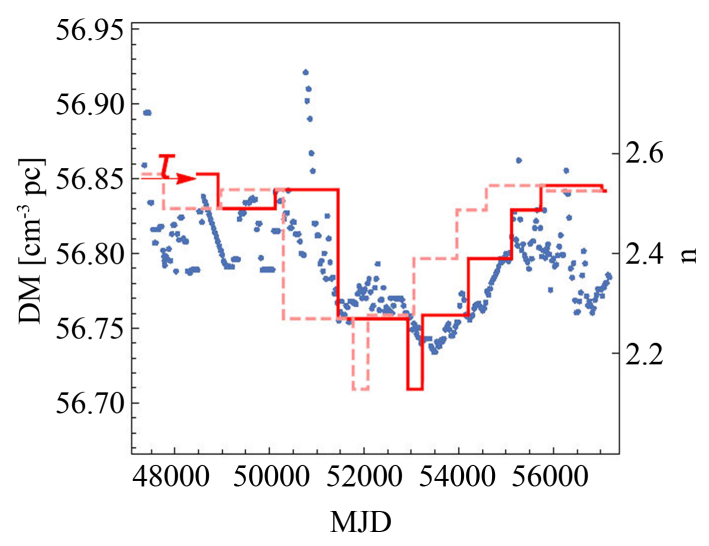

Figure 4. The relationship between the dispersion measure $D M$ (blue dots) and the braking index $n$ (red dashed line, horizontal segments). The vertical segments of the dashed line record the glitches. The solid red line shows the braking index with a shift of 1010 days. MJD $=$ JD $-2,400,000$. The correlation coefficient between the dispersion measure and the braking index is 0.7 .

increase in the dispersion measure. Intermediary models have been discussed by Kou and Tong [27]. They consider that glitches are related to changes in interior of the neutron stars, but may lead to some effect in the outer magnetosphere. In turn, plasma instabilities in the nebula can disrupt the configuration of magnetic field lines, when fields of opposite polarity are pressed together, and cause gamma-ray flare, similar to flares in the solar corona. The same opinion says Buhler and Blangdford [1]: "The gamma-ray flares are therefore likely connected to explosive reconnection events triggered by current instabilities". Striany et al. also support this idea [28], but they note: "However, evidence for magnetic field reconnection events in the Crab Nebula is elusive, and no optical or X-ray emission in coincidence with the gamma-ray flaring has been unambiguously detected to date (e.g., Weisskopf et al. 2012)". According to our data (Figure 3) gamma-flares have tendency to concentrate during the period of enhanced disturbances in the Crab Nebula, that can be considered as support of this model. As for the correlation between of pulsar glitches and gamma-ray flares Buhler and Blandford [1] write: "The time scale of the recurrence of pulsar glitches is similar to the recurrence of the gamma-ray flares, however, there is no obvious correlation in time between these two events".

\section{Conclusions}

The scattering of the Crab pulsar radio emission at a frequency of $111 \mathrm{MHz}$ according to our measurements and the dispersion measure provided in Jodrell Bank, show secular variations they are well correlated (correlation coefficient $0.80 \pm 0.05)$.

The observed changes in the pulsar pulse scattering and the dispersion measure and their partial correlation are explained by the passage of plasma clouds in front of the pulsar with a size of about $0.92 \times 10^{-4} \mathrm{pc}\left(3.0 \times 10^{14} \mathrm{~cm}\right)$ with varying degrees of turbulence. 
In 2002-2007, during the quiet period, in the Crab Nebula $C_{n}^{2}=0.0115 \pm$ $0.0038 \mathrm{~m}^{-7}$ and $\Delta n_{e} / n_{e} \sim 0.01$.

In 2008-2013, during the active period, in the Crab Nebula $C_{n}^{2}=0.115 \pm$ $0.038 \mathrm{~m}^{-7}$ and $\Delta n_{e} / n_{e} \sim 0.03$.

Turbulence in the disturbed region is higher than in quiet ones and much be higher than in the interstellar medium.

Various types of pulsar radio emission irregularities can be explained by physical processes occurring in the pulsar's magnetosphere. Perturbations in the pulsar's magnetosphere can lead to ejection of charged particles into the Nebula, lead to instability of the plasma, disrupt the configuration of the magnetic field lines and cause gamma-ray flares. According to our data gamma-flares have tendency to take place during the period of enhanced disturbances in the Crab Nebula that can be considered as support of this model. New observations are needed to support this hypothesis.

\section{Funding}

This work was supported by the research program of the Presidium of the Russian Academy of Sciences "Non-stationary phenomena in the objects of the Universe"

\section{Acknowledgements}

The author thanks the staff of the meter wave-length technical service laboratory of the Pushchino Radio Astronomy Observatory for the providing reliable observations. The author is very thankful to Managing Editor of IJAA Jane Gao for the fruitful cooperation and the anonymous reviewers for valuable notices.

\section{Conflicts of Interest}

The author declares no conflict of interest, regarding the publication of this paper.

\section{References}

[1] Buhler, R. and Blandford, R. (2014) The Surprising Crab Pulsar and Its Nebula: A Review. Reports on Progress in Physics, 77, Article ID: 066901. https://doi.org/10.1088/0034-4885/77/6/066901

[2] Staelin, D.H. and Reifenstein, E.C. (1968) Pulsating Radio Sources near the Crab Pulsar Nebula. Science, 162, 1481-1483.

https://doi.org/10.1126/science.162.3861.1481

[3] Rankin, J.M. and Counselman, C.C. (1973) Pulsar NP 0532: Variability of Dispersion and Scattering. The Astrophysical Journal, 181, 875-889.

https://doi.org/10.1086/152099

[4] Isaacman, R. and Rankin, J.M. (1977) The Crab Nebula Pulsar: Variability of Dispersion and Scattering. The Astrophysical Journal, 214, 214-232. https://doi.org/10.1086/155245

[5] Kuz'min, A.D., Belyatsky, Yu.A., Dumsky, D.V., Izvekova, V.A., Lapaev, K.A., Log- 
vinenko, S.V., Losovsky, B.Ya. and Pugachev, V.D. (2012) Monitoring of the Radio Emission of the Crab Nebula Pulsar at Low Frequencies. Astronomy Reports, 55, 416-424. https://doi.org/10.1134/S1063772911040032

[6] Losovsii, B.Ya. (2017) The Dynamics and Structure of Plasma Inhomogeneities in the Crab Nebula. Astronomy Reports, 61, 187-192.

https://doi.org/10.1134/S1063772917030052

[7] Losovsky, B.Ya., Dumsky, D.V. and Belyatsky, Yu.A. (2019) Relationship between Scattering and Dispersion Measure of the Crab Nebula Pulsar. Astronomy Reports, 63, 830-834. https://doi.org/10.1134/S1063772919090051

[8] Alurkar, S.K., Slee, O.B. and Bobra, A.D. (1986) Scattering of Pulsar Radiation and Electron Density Turbulence in the Interstellar Medium. Australian Journal of Physics, 39, 433-448. https://doi.org/10.1071/PH860433

[9] Kuzmin, A., Losovsky, B.Ya., Jordan, C.A. and Graham-Smith, F. (2008) Correlation of the Scattering and Dispersion Events in the Crab Nebula Pulsar. Astronomy \& Astrophysics, 483, 13-14. https://doi.org/10.1051/0004-6361:20079211

[10] Manchester, R. (1977) Taylor J. Pulsars. Freeman, San Francisco; Moscow, 1980.

[11] Abdo, A.A., Ackermann, M., Ajello, M., et al. (2011) Gamma-Ray Flares from the Crab Nebula. Science, 331, 739-755.

[12] Ojha, R., Hays, E., Buehle, R. and Dutka, M. (2013) Fermi LAT Detection of a New Gamma-Ray Flare from the Crab Nebula Region. Atel, 4855.

[13] Buehler, R., Scargle, J.D., Blandford, R.D., et al. (2012) Gamma-Ray Activity in the Crab Nebula. The Exceptional Flare of 2011 April. The Astrophysical Journal, 749, 26. https://doi.org/10.1088/0004-637X/749/1/26

[14] Tavani, M., Bulgarelli, A., Vittorini, V., et al. (2011) Discovery of Powerful Gamma-Ray Flares from the Crab Nebula. Science, 331, 736-759.

[15] Striani, E., Tavani, M., Vittorini, V., et al. (2013) Variable Gamma-Ray Emission from the Crab Nebula: Short Flares and Long "Waves". The Astrophysical Journal, 765, 52S. https://doi.org/10.1088/0004-637X/765/1/52

[16] Bulgarelli, A., Tavani, M., Parmiggiani, N., et al. (2018) AGILE Detection of Renewed Gamma-Ray Activity from Crab Nebula. Atel, 12148.

[17] Huang, X., Yuan, Q. and Fan, Y. (2021) Ray Flares from the Crab Nebula with Fermi-LAT. The Astrophysical Journal, 908, 65.

[18] McKee, J.W., Lyne, A.G., Stappers, B.W., Bassa, C.G. and Jordan, C.A. (2018) Temporal Variations in Scattering and Dispersion Measure in the Crab Pulsar and Their Effect on Timing Precision. Monthly Notices of the Royal Astronomical Society, 479, 4216-4224. https://doi.org/10.1093/mnras/sty1727

[19] Kuzmin, A.D. and Losovsky, B.Ya. (1999) Observations of the Mean Profiles of Radio Pulsar and the Structure of Their Emitting Regions at $102 \mathrm{MHz}$. Astronomy Reports, 43, 288-304.

[20] Cordes, J.M., Weisberg, J.M. and Boriakoff, V. (1985) Small-Scale Electron Density Turbulence in the Interstellar Medium. The Astrophysical Journal, 288, 221-247. https://doi.org/10.1086/162784

[21] Kuzmin, A.D., Kondrat'ev, V.I., Kostyuk, S.V., Losovsky, B.Ya., Popov, M.V., Soglasnov, V.F., D'Amico, N. and Montebugnoli, S. (2002) Frequency Dependence of the Scattering Pulse Broadening for Crab Pulsar. Astronomy Letters, 28, 251-256. https://doi.org/10.1134/1.1467260

[22] Yao, J.M., Manchester, R.N. and Wang, N. (2017) A New Electron-Density Model for Estimation of Pulsar and FRB Distances. The Astrophysical Journal, 835, 29-59. https://doi.org/10.3847/1538-4357/835/1/29 
[23] Kuzmin, A.D., Losovsky, B.Ya., Logvinenko, S.V. and Litvinov, I.I. (2008) Deviation of the Arrival-Time Delay of Pulses from the Crab Pulsar from the Quadratic Frequency Relation. Astronomy Reports, 52, 910-916.

https://doi.org/10.1134/S106377290811005X

[24] Espinoza, C.M., Lyne, A.G., Stappers, B.W. and Kramer, M. (2011) A Study of 315 Glitches in the Rotation of 102 Pulsars. Monthly Notices of the Royal Astronomical Society, 414, 1679-1704. https://doi.org/10.1111/j.1365-2966.2011.18503.x

[25] Cadez, A., Zampiery, L., Barbiery, C., Calvani, M., Naletto, G., Barbiery, M. and Ponikwar, D. (2016) What Brakes the Crab Pulsar. Astronomy \& Astrophysics, 587, A99. https://doi.org/10.1051/0004-6361/201526490

[26] Lyne, A.G., Jordan, C.A., Graham-Smith, F., Espinoza, C.M., Stappers, B.W. and Weltevrede, P. (2015) 45 Years of the Rotation of the Crab Pulsar. Monthly Notices of the Royal Astronomical Society, 446, 857-864. https://doi.org/10.1093/mnras/stu2118

[27] Kou, F.F. and Tong, H. (2015) Rotational Evolution of the Crab Pulsar in the Wind Braking Model. Monthly Notices of the Royal Astronomical Society, 450, 1990-1998. https://doi.org/10.1093/mnras/stv734

[28] Striani, E., Tavani, M., Vittorini, V., et al. (2013) Variable Gamma-Ray Emission from the Crab Nebula: Short Flares and Long "Waves". The Astrophysical Journal, 765, 52-62. https://doi.org/10.1088/0004-637X/765/1/52 